\title{
Efeito de um Programa de Histórias com Abordagem Sociocognitiva em Crianças de Educação Infantil
}

\author{
Jaqueline Pereira Dias-Corrêa ${ }^{1}$ \\ Faculdade Anhanguera de São José dos Campos \\ Edna Maria Marturano \\ Universidade de São Paulo \\ Marisa Cosenza Rodrigues \\ Universidade Federal de Juiz de Fora \\ Andressa Kutschenko Nahas \\ Universidade de São Paulo
}

\begin{abstract}
RESUMO - Este estudo objetivou avaliar um programa para promoção de habilidades sociocognitivas, baseado na leitura de histórias, quanto a possíveis efeitos no desenvolvimento sociocognitivo e comportamental. O programa conta com 25 livros infantis, ricos em pistas sociais. Participaram duas turmas consecutivas da educação infantil, compondo dois grupos, GI e GII. Para aferir efeitos do programa, foram avaliadas habilidades sociocognitivas, habilidades sociais e problemas de comportamento. GI foi avaliado antes e depois de passar pelo programa. No ano seguinte, GII foi avaliado antes da intervenção duas vezes, com intervalo equivalente ao da duração do programa, e novamente depois da intervenção. Comparações entre e intra grupos mostraram aumento nas habilidades sociocognitivas e sociais, bem como redução de dificuldades comportamentais, sugerindo efeitos positivos do programa.
\end{abstract}

Palavras-chaves: educação infantil, cognição social, intervenção psicológica

\section{Effect of a Program of Stories with a Social Cognitive Approach in Kindergarten Children}

\begin{abstract}
This study aimed to evaluate a story reading program for possible effects on children's socio-cognitive and behavioral development. The program is comprised by 25 storybooks, which are rich in social cues. It was administered to two consecutive classes of early childhood education, which compound two groups, GI and GII. To investigate effects of the program, socio-cognitive skills, social skills, and behavior problems were assessed. GI was evaluated before and after the intervention. During the following year, GII was assessed twice before intervention, with an interval equal to the duration of the program, and again after the intervention. Comparisons between and within groups showed an increase in socio-cognitive and social skills, and reduced behavioral difficulties, suggesting positive effects of the program.
\end{abstract}

Keywords: early childhood education, social cognition, psychological intervention

A educação infantil é a primeira etapa de escolarização da criança. A entrada na escola é um marco que atualiza e amplia suas interações sociais (Borges \& Marturano, 2009). Atualmente, as crianças têm cada vez mais cedo seus primeiros contatos sociais fora do ambiente familiar. $\mathrm{O}$ contexto escolar desempenha, portanto, um papel relevante na socialização da criança (Mayeux \& Cillessen, 2003; Thomas, Bierman, \& Powers, 2011). Brownell et al. (2008) ressaltam que as experiências positivas das crianças com seus companheiros em espaços coletivos de socialização nos anos pré-escolares são preditoras da competência social. Em contrapartida, as dificuldades relacionais nos anos da educação infantil predizem trajetórias desfavoráveis no ensino fundamental (Silver, Measelle, Armstrong, \& Essex, 2010).

Crianças que já na pré-escola mostram menor competência social ou altos níveis de comportamento externalizante,

1 Endereço para correspondência: Rua Teresina, 494, apartamento 43, Parque Industrial, São José dos Campos, SP, Brasil. CEP. 12235-610.

E-mail: jaquepdias@gmail.com como agressividade, agitação, impulsividade, características antissociais e desafiantes (Achenbach \& Edelbrock, 1979), estão em risco de dificuldades adaptativas mais tarde, na adolescência (Berlin, Dunning, \& Dodge, 2011; BolsoniSilva, Marturano, \& Freiria, 2009; Bornstein, Hahn, \& Haynes, 2010; Burt \& Roisman, 2010). Nesse cenário, considera-se que as habilidades sociais têm efeito protetor no desenvolvimento, por promoverem relacionamentos positivos com os pares e prevenirem comportamentos agressivos (Del Prette \& Del Prette, 2005).

A escola, ambiente essencialmente interativo, que estabelece regras de convívio social, é um espaço potencial para se prevenirem problemas de comportamento (Borges \& Marturano, 2009; Rodrigues, Dias, \& Freitas, 2010) e promover desenvolvimento saudável. Além disso, as crianças de educação infantil estão particularmente sensíveis à estimulação (Lansford et al., 2006); assim, maximizar potencialidades no contexto da pré-escola pode influenciar de forma decisiva a trajetória futura. Nessa perspectiva, promover desenvolvimento visando ao aprimoramento de 
forças adaptativas, solução para os problemas cotidianos, capacidade de enfrentamento das adversidades e interesse por si e pelo mundo social (Rodrigues, 2008) torna-se uma estratégia atual e promissora.

\section{Promovendo o Desenvolvimento Sociocognitivo}

O desenvolvimento sociocognitivo envolve o conhecimento e a compreensão do mundo social (Rodrigues, Oliveira, Rubac, \& Tavares, 2007); caracteriza o pensamento sobre as pessoas, sobre o que elas fazem ou deveriam fazer e sobre como elas pensam e sentem. A cognição social compreende os processos e mecanismos cognitivos envolvidos nos fenômenos psicológicos sociais e é aprimorada com o desenvolvimento da criança (Yamaguchi, Kuhlmeier, Wynn, \& vanMarle, 2009).

No âmbito da cognição social, inserem-se modelos denominados de processamento de informação social, os quais concebem a mente humana como um sistema que manipula e transforma as informações advindas das situações interativas e sociais mais amplas (Dodge \& Crick, 1994). Os modelos de processamento da informação com foco nas informações sociais buscam compreender como os acontecimentos psicológicos sociais são processados e organizados; descrevem os processos cognitivos relacionados a uma resposta comportamental, adaptada ou não, a um estímulo social, tais como: resposta agressiva a uma provocação, resposta depressiva a uma rejeição (Dodge \& Crick, 1994; Helmsen, Koglin, \& Petermann, 2012; Teglasi \& Rothman, 2001). Por meio desses modelos, é possível compreender como as informações comportamentais são transformadas em memória e como orientam o processamento futuro. Também é possível compreender os mecanismos envolvidos nos processos sucessivos de codificação da informação, interpretação do comportamento e regulação das emoções, assim como na concepção, seleção e emissão de uma resposta.

Vasconcellos, Picon, Prochnow e Gauer (2006), em um estudo de revisão de literatura, afirmam que, entre os modelos de processamento da informação social que visam compreender os mecanismos da agressão infantil, o modelo de Dodge (1986) foi o mais robusto, ou seja, evidenciou mais resultados empíricos. O pesquisador, baseado em dados empíricos sobre o modo como crianças e adolescentes agressivos processam as informações sociais, elaborou um modelo de processamento da informação (Social Information Processing [SIP]), propondo que as atitudes infantis são permeadas por quatro passos mentais: codificação das pistas sociais; interpretação das pistas sociais; acesso à memória, recuperando informações já armazenadas e avaliação da possível resposta; seleção da resposta.

Dodge e Crick (1994), analisando criticamente o modelo, concluíram ser necessária sua reformulação e ampliação. Essa ampliação permitiu compreender de forma mais acurada os mecanismos que contribuem para o comportamento socialmente competente e o ajustamento social infantil, bem como os comportamentos relacionados à agressão e ao comportamento antissocial. O modelo reformulado, que prevê uma estrutura cíclica de processamento e a influencia das emoções, propõe seis passos mentais: (a) codificação das pistas internas e externas; (b) interpretação e representação mental dessas pistas sociais; (c) clarificação ou seleção de um objetivo; (d) construção ou clarificação da resposta; (e) resposta/decisão; e (f) emissão da resposta. O modelo vem sendo bastante discutido e investigado (Helmsen et al., 2012; Lansford et al., 2006).

Lansford et al. (2006) apontam que, entre a educação infantil e os primeiros anos do ensino fundamental, as crianças não apresentam padrões estáveis de processamento da informação social. Tal conclusão, que denota ser essa uma capacidade maleável, passível de modificação, abre espaço para intervenções precoces que promovam o desenvolvimento, a ampliação e o aprimoramento de habilidades sociocognitivas (Fraser et al., 2005; Helmsen et al., 2012; Mayeux \& Cillessen, 2003; Rodrigues, 2014).

A promoção das habilidades sociocognitivas visa fomentar o desenvolvimento, refinamento e aprimoramento da compreensão do mundo social (Rodrigues \& Ribeiro, 2011). O aprimoramento sociocognitivo, por meio de situações que estimulem a criança a observar, pensar e avaliar as possíveis consequências antes de emitir uma resposta comportamental, tende a contribuir para o ajustamento social e o desenvolvimento infantil (Rodrigues \& Ribeiro, 2011). Programas de desenvolvimento sociocognitivo têm sido propostos para promoção das habilidades de resolução de problemas (Borges \& Marturano 2009; Rodrigues et al., 2010), promoção da empatia (Rodrigues \& Ribeiro, 2011), aprimoramento da competência social e redução dos comportamentos desadaptativos (Berlin et al., 2011; Fraser et al., 2005; Teglasi \& Rothman, 2001).

Uma ferramenta útil, porém pouco explorada no contexto brasileiro, utilizada como recurso promotor sociocognitivo e para a prevenção de comportamentos antissociais e agressivos são os livros de histórias infantis (Kalyva \& Agaliotis, 2009; Rodrigues \& Ribeiro, 2011; Rodrigues, 2014; Teglasi \& Rothman, 2001). Os livros de histórias possibilitam a interpretação dos acontecimentos que envolvem os personagens por meio de pistas sociais externas, presentes em eventos e circunstâncias, bem como pistas internas, associadas a sentimentos, metas, desejos (Rodrigues, 2008; Teglasi \& Rothman, 2001). A exploração dos acontecimentos, pensamentos e sentimentos através de uma experiência emocional, inserida em uma história infantil, tende a promover uma reflexão por parte da criança quanto aos próprios pensamentos, sentimentos e atitudes (Borges \& Marturano, 2009; Teglasi \& Rothman, 2001).

Teglasi e Rothman (2001) realizaram uma intervenção, embasada no modelo ampliado de processamento da informação social de Dodge e Crick (1994), em contexto escolar, utilizando a literatura infantil para promover o desenvolvimento sociocognitivo de crianças agressivas. $\mathrm{O}$ programa conta com seis componentes que visam explorar e discutir o conteúdo da história, o que possibilita à criança maior compreensão e um aprimoramento do processamento das informações sociais. O primeiro componente é: o que está acontecendo? As crianças observam e interpretam as pistas sociais presentes na cena e identificam o problema da história. As ilustrações são exploradas em termos de posturas e expressões faciais dos personagens. O segundo 
componente é: o que os personagens estão pensando e sentindo? As crianças são incentivadas a fazer inferências sobre o "mundo interno" dos personagens, relacionando sentimentos e desejos, bem como as interpretações deles para com os acontecimentos da história. O terceiro componente se traduz na seguinte pergunta: quais são as intenções e metas dos personagens? Discutem-se com as crianças os objetivos particulares dos personagens à luz dos pensamentos e sentimentos presentes na história. Há, também, a reflexão quanto à avaliação das metas e intenções presentes na história. A pergunta correspondente ao quarto componente é: o que os personagens alcançam com suas ações? Discutese com as crianças a relação entre os objetivos e metas dos personagens e os resultados alcançados por eles, levando em consideração o quanto as emoções influenciam a tomada de decisão. Em relação ao quinto componente - como os personagens executam e monitoram seus comportamentos? -, as crianças identificam as razões dos personagens para a emissão de um comportamento e avaliam as consequências advindas dessas ações; assim, as crianças refletem sobre o êxito ou insucesso do comportamento emitido pelo personagem. No sexto e último componente - quais as lições aprendidas? -, a criança, por meio das experiências e resolução de problemas vivenciados pelos personagens, tem a oportunidade de ampliar suas experiências, estabelecendo relações com aquelas vivenciadas em seu dia a dia.

Os resultados encontrados por Teglasi e Rothman (2001), com crianças de $4^{\mathrm{a}}$ e $5^{\mathrm{a}}$ série do ensino fundamental de uma escola estadunidense, foram promissores. Quando comparados a um grupo controle, os grupos expostos ao programa apresentaram redução significativa da agressividade, com diminuição de condutas antissociais e comportamentos externalizantes nas crianças identificadas como não-agressivas e entre aquelas identificadas como agressivas.

Rodrigues (2014) descreve intervenção em grupo semelhante, que objetiva prevenir a agressividade e promover habilidades sociocognitivas por meio das histórias infantis em crianças de 6 a 10 anos com dificuldades de adaptação social, tais como agressividade, comportamento antissocial, isolamento, rejeição e timidez. A intervenção, conduzida em serviço-escola de psicologia, é baseada no programa elaborado por Teglasi e Rothman (2001), anteriormente descrito. A seleção dos livros de histórias, ricos em pistas sociais, fundamenta-se em análise prévia de livros de histórias infantis nacionais (Rodrigues et al., 2007). Como informa Rodrigues (2014), os resultados dessa proposta extensionista apontam para uma direção positiva no que tange ao aprimoramento do processamento de informação social das crianças e benefícios no que se refere ao relacionamento interpessoal, com a minimização do comportamento agressivo dos participantes.

Sendo assim, constata-se, no plano aplicado, a viabilidade do uso de livros infantis como recurso promotor de desenvolvimento sociocognitivo e prevenção de comportamentos agressivos e antissociais (Rodrigues, 2014; Teglasi \& Rothman, 2001). Nessa perspectiva, o presente estudo, inspirado nas intervenções realizadas por Rodrigues (2014), objetiva avaliar o efeito de um programa, previamente elaborado, com base na leitura de histórias infantis no desenvolvimento sociocognitivo e comportamental de crianças inseridas na última etapa da educação infantil.

\section{Método}

A pesquisa foi realizada em uma escola de Educação Infantil, escolhida por conveniência ${ }^{1}$, situada em município paulista. Trata-se de instituição educativa mantida por uma Organização não Governamental.

\section{Delineamento}

Foi adotado um delineamento de comparação de grupos, com avaliação pré e pós-intervenção. Um dos grupos, GI, foi avaliado duas vezes: antes e depois da intervenção. $\mathrm{O}$ outro, GII, foi avaliado três vezes. As duas primeiras avaliações de GII ocorreram antes da intervenção, em momentos separados por intervalo de tempo equivalente ao que separou as avaliações de GI. Logo após a intervenção, GII foi novamente avaliado. Esse plano teve a finalidade de possibilitar a verificação da eficiência da intervenção com relação ao avanço cronológico natural das habilidades e comportamentos avaliados.

\section{Participantes}

Participaram 45 crianças, alunas de duas turmas de PréIII (atual Etapa II), distribuídas em dois grupos: GI - Grupo participante da pesquisa em 2010, constituído pelos 25 alunos de uma classe, sendo 17 meninos e 8 meninas, com idade variando, no início da intervenção, entre 68 meses e 79 meses (média 73 meses); GII - Grupo participante da pesquisa em 2011, constituído pelos 20 alunos de uma classe, sendo 12 meninos e 8 meninas, com idade variando entre 60 e 75 meses (média 67 meses) no início da coleta, e entre 63 e 78 meses (média 70 meses) no início da intervenção. Uma criança do GII saiu da escola, de modo que, na última avaliação, esse grupo contava com 19 participantes. Participaram ainda duas professoras, como informantes da pesquisa. Elas se alternavam na condução das turmas, nos turnos matutino e vespertino.

O estudo foi aprovado por Comitê de Ética em Pesquisa. Os responsáveis pelos participantes assinaram o Termo de Consentimento Livre e Esclarecido, autorizando as crianças a participarem da pesquisa.

\section{Materiais e Instrumentos}

Para a intervenção, foram utilizados 25 livros de histórias infantis, ricos em pistas sociais, internas e externas. Esse material foi selecionado de uma lista de 100 livros analisados

1 A primeira autora do presente estudo foi apresentada à assistente social da escola pela segunda autora, orientadora do projeto. A assistente social, por sua vez, mediou o primeiro contato com a diretora 
do ponto de vista do processamento da informação social em pesquisa precedente (Rodrigues et al., 2007).

A coleta de dados contou com três instrumentos: a Avaliação Sociocognitiva de Respostas Infantis PósExploração Dialogada de Narrativa Textual, elaborada por Rodrigues (2014) e aferida por Dias (2012); o Questionário de Respostas Socialmente Habilidosas Segundo Relato do Professor (QRSH-RP; Bolsoni-Silva, Marturano, \& Loureiro, 2009) e o Questionário de Capacidades e Dificuldades versão do professor (SDQ; Fleitlich, Cartázar, \& Goodman, 2000).

Para a avaliação sociocognitiva das crianças, utilizou-se o livro Anjinho de Eva Furnari (2004). O enredo do livro refere-se a um anjinho que, ao deixar cair seu sapato na Terra, vai à procura dele sem permissão de sua mãe. Na Terra, lugar onde nunca havia ido antes, vê vários monstros, o que o faz sentir muito medo. Depois de um tempo procurando o sapato e fugindo dos monstros, o anjinho reencontra a mãe com seu sapato e seus óculos. Sua mãe então coloca os óculos em seu filho e lhe mostra que o que ele via não eram monstros, eram apenas objetos e pessoas. O livro em questão é lido para a criança de forma mediada, de modo que as intervenções do examinador suscitem a reflexão do examinado sobre os seis componentes propostos por Teglasi e Rothman (2001). O desempenho da criança é avaliado por meio de escalas tipo Likert: (1) Muito bom, (2) Bom, (3) Regular, (4) Fraco, (5) Muito fraco, (6) Ausente.

$\mathrm{Na}$ intervenção, lançou-se mão, no presente estudo, do mesmo livro utilizado por Rodrigues (2014) e o procedimento de aplicação e correção proposto por Dias (2012). Esse procedimento inclui um roteiro com 114 perguntas sobre a história e um guia de correção, com critérios para avaliação das respostas da criança a 80 perguntas do roteiro, agrupadas em seis componentes (Teglasi \& Rothman, 2001): O que está acontecendo? O que os personagens estão pensando e sentindo? Quais as metas e intenções? O que os personagens alcançam com suas ações? Como executam e monitoram comportamentos? Quais as lições aprendidas? A avaliação pode variar de muito fraca a muito boa, em uma escala de 5 pontos: $1=$ muito fraca, $2=$ fraca, $3=$ regular, $4=$ boa e $5=$ muito boa. Os escores são somados e fornecem um escore para cada componente, assim como o escore total da criança avaliada. Dias (2012) reporta confiabilidade satisfatória do procedimento de avaliação das respostas, aferida por meio de testes de concordância entre avaliadores. Os índices de correlação entre avaliadoras ( $r$ de Pearson) variaram entre 0,83 e 0,95 .

O QRSH-RP investiga as habilidades sociais de crianças pré-escolares em 24 itens descritivos de comportamentos socialmente habilidosos. Para cada item, há uma escala de três pontos $(2=$ se aplica, $1=$ se aplica em parte, $0=$ não se aplica). Os escores são somados e fornecem o escore total da criança avaliada. O QRSH-RP se estrutura em três fatores: Sociabilidade e Expressividade Emocional, com 14 itens; Iniciativa Social, com seis itens; e Busca de Suporte, com três itens. No que tange às propriedades psicométricas do instrumento, tem-se consistência interna satisfatória (valores de alfa entre 0,73 e 0,92 para os fatores) e indícios de validade de construto, discriminante e preditiva, indicando que o questionário está aferido para a avaliação de respostas socialmente habilidosas de pré-escolares (Bolsoni-Silva et al., 2009a).

O SDQ (Goodman, 1997) visa investigar comportamentos adequados e não adequados em crianças e adolescentes, na faixa etária de 4 a 16 anos e foi adaptado para o Brasil (Fleitlich et al., 2000). Conta com 25 itens agrupados em cinco escalas, cada qual com cinco itens descritivos de comportamentos: Sintomas Emocionais, Problemas de Conduta, Hiperatividade/Déficit de Atenção, Problemas de Relacionamento e Comportamento Prossocial. A avaliação de cada item é feita em uma escala de três pontos: $0=$ falso, $1=$ mais ou menos verdadeiro, $2=$ verdadeiro. $\mathrm{O}$ instrumento apresenta satisfatória consistência interna, $\operatorname{com} \alpha=0,82$ para o total das dificuldades; 0,75 para os sintomas emocionais; 0,72 para os problemas de comportamento; 0,69 para a hiperatividade; 0,65 para o comportamento prossocial; e 0,61 para os problemas de relacionamento com os colegas.

\section{Procedimentos}

Antes da coleta de dados, em 2010, fez-se um breve treinamento com as duas professoras regentes da turma no que tange ao preenchimento dos instrumentos QRSH-RP e SDQ. Em agosto de 2010, em encontro com ambas as professoras, a primeira autora repassou as instruções de preenchimento e esclareceu possíveis dúvidas. A professora do turno matutino avaliou 13 crianças e a do turno vespertino, 12 crianças.

Para a sondagem das habilidades sociocognitivas, realizada pela primeira autora do presente estudo, as crianças foram avaliadas individualmente durante o horário de aula, em sala cedida pela direção da escola. A história Anjinho era lida para a criança, de forma mediada, com base no roteiro de perguntas. A avaliação era gravada.

Após as avaliações, procedeu-se à composição de subgrupos para a intervenção no GI. Foram formados três subgrupos, de modo a não ultrapassar nove crianças por grupo (Teglasi \& Rothman, 2001), uma vez que o programa demanda discussões e reflexões acerca de cada história contada na sessão. As crianças foram agrupadas com a ajuda das professoras, com a finalidade de garantir a semelhança entre os subgrupos, de modo que crianças tímidas, agitadas, participativas, hostis fossem alocadas em cada um.

A intervenção no GI foi feita com periodicidade de duas a três sessões de $50 \mathrm{~min}$. por semana, de agosto a novembro de 2010, totalizando 25 sessões. Inicialmente, cada sessão era organizada em três partes: (a) elaboração e/ou recordação das regras visando à regulação da conduta; (b) contação mediada da história com base nos componentes propostos por Teglasi e Rothman (2001); (c) atividades lúdicas complementares à história com o objetivo de consolidar o aprendizado das crianças. O procedimento (c) se estendeu até a $10^{\mathrm{a}}$ sessão; fez-se necessário retirá-lo das demais sessões, uma vez que estas estavam demandando maior reflexão da história, assim o tempo estava sendo todo ocupado com as discussões e reflexões. Ao final da intervenção, as crianças foram reavaliadas.

A reavaliação, denominada de pós-intervenção, seguiu os mesmos procedimentos da pré-intervenção. Cada professora 
reavaliou as mesmas crianças avaliadas na pré-intervenção. As avaliações sociocognitivas foram conduzidas da mesma maneira, as crianças foram avaliadas individualmente pela pesquisadora. A pesquisadora realizou, também, uma reunião com os pais das crianças participantes, ao final da intervenção, a fim de repassar as impressões gerais do estudo e incentivar a leitura de histórias em contexto familiar.

No ano de 2011, o estudo seguiu os mesmos procedimentos de avaliação e implementação do programa. As mesmas professoras permaneceram responsáveis pela nova turma do Pré III, que formou o GII. De acordo com o delineamento do estudo, em 2011, houve a inserção de um novo momento avaliativo denominado de pré-intervenção 1. Tal avaliação possibilita, nas análises estatísticas, a aferição de efeitos de medidas repetidas, como, por exemplo, uma possível tendência do professor para avaliar mais positivamente o aluno em cada nova avaliação, ou um possível efeito de aprendizagem da criança como consequência de ser exposta repetidamente ao mesmo procedimento de exploração da história. As crianças que compuseram o GII foram avaliadas, portanto, em três momentos: pré-intervenção 1, pré-intervenção 2 e pós-intervenção.

A avaliação pré-intervenção 1 , realizada em maio de 2011, seguiu os mesmos procedimentos das avaliações realizadas no ano anterior. Após três meses, intervalo de tempo correspondente ao da duração da intervenção, foi feita a avaliação pré-intervenção 2, em agosto de 2011.

A implementação do programa seguiu os mesmos passos da intervenção realizada em 2010, iniciada em agosto e findada em novembro. O GII foi subdivido em três grupos. O programa foi desenvolvido de modo similar ao de 2010 , seguindo, assim, o mesmo número de sessões, tempo de duração e demais procedimentos.

Em novembro de 2011, foi realizada a avaliação pósintervenção nas crianças do GII. Realizou-se, também, uma reunião com os pais das crianças, seguindo os mesmos propósitos daquela realizada em 2010.

\section{Resultados}

Os grupos foram comparados quanto à idade, por meio do teste $t$. Não se encontrou diferença entre as médias de GI e GII no início da intervenção $(t=1,094 ; p=0,280)$. Para as análises de dados, foi utilizado o modelo linear de efeitos mistos (efeitos aleatórios e fixos), em que as respostas de um mesmo indivíduo estão agrupadas e a suposição de independência entre as observações em um mesmo grupo não é adequada (Schall, 1991). Para a utilização desse modelo, é necessário que seus resíduos tenham distribuição normal com média zero e variância constante. $\mathrm{O}$ ajuste do modelo foi feito através do procedimento PROC MIXED, do software $\mathrm{SAS} \AA$ 9.2.

Na avaliação pré-intervenção (para GII, pré-intervenção 2), o teste t indicou que GI e GII eram equivalentes, exceto para a variável $O$ que está acontecendo?, em que houve diferença estatística significativa a favor de GII $(t=2,15$ $p=0,036)$. Portanto, GI e GII não apresentaram equivalência para essa variável ao iniciarem a intervenção.
$\mathrm{Na}$ avaliação pós-intervenção, GI e GII mostraram equivalência entre eles em todas as variáveis de interesse do estudo, exceto para o fator Iniciativa Social do instrumento QRSH-RP. O resultado encontrado para esse fator evidencia diferença estatística significativa entre os grupos $(t=1,57$ $p=0,05$ ), indicando que GII terminou o programa de histórias infantis apresentando melhor resultado nessa variável.

A Tabela 1 apresenta os resultados encontrados para o instrumento Avaliação Sociocognitiva de Respostas Infantis Pós-Exploração Dialogada de Narrativa Textual, com a pontuação média dos participantes em cada componente do instrumento em dois momentos para o GI e em três momentos para o GII, de acordo com o delineamento da pesquisa. $\mathrm{Na}$ Tabela 1, também se apresentam os resultados da análise estatística das diferenças obtidas entre as avaliações consecutivas, separadamente nos dois grupos.

Para GI, encontrou-se aumento significativo nas médias entre as avaliações pré e pós-intervenção, em todos os componentes e no escore total do instrumento. Para GII, encontrou-se aumento significativo já entre as avaliações pré-intervenção 1 e pré-intervenção 2 , nas médias dos componentes $O$ que está acontecendo? e $O$ que os personagens estão pensando e sentindo?, bem como no escore total. Na comparação entre as avaliações préintervenção 2 e pós-intervenção, GII apresentou resultados semelhantes a GI, ou seja, aumento nas médias dos seis componentes e do escore total.

$\mathrm{Na}$ Tabela 2, encontram-se os resultados do QRSH-RP, com a pontuação média dos participantes em cada fator do instrumento e os resultados da análise estatística das diferenças entre avaliações consecutivas de GI e GII. Os resultados das comparações entre as avaliações pré e pósintervenção em GI indicaram aumento significativo nas médias do escore total do instrumento e dos três fatores: Sociabilidade e Expressividade Emocional, Busca por Suporte e Iniciativa Social.

Em GII, observou-se aumento significativo na média do fator Iniciativa Social entre os momentos pré-intervenção 1 e pré-intervenção 2. Na comparação entre as avaliações préintervenção 2 e pós-intervenção, houve aumento significativo no escore total do instrumento e nos três fatores.

Os resultados para o instrumento SDQ são apresentados na Tabela 3. Nessa tabela, são apresentadas as médias dos grupos nas classes de comportamento e os resultados da análise estatística referente às diferenças obtidas entre avaliações consecutivas para GI e GII separadamente.

Para GI, as escalas Hiperatividade, Problemas de Relacionamento e o escore total de dificuldades apresentaram uma redução estatisticamente significativa nas comparações entre avaliação pré e pós-intervenção; ainda nesse grupo, encontrou-se um aumento significativo para Comportamento Prossocial. Para GII, não foram encontradas diferenças entre pré-intervenção 1 e pré-intervenção 2 ; em contrapartida, nas comparações entre pré-intervenção 2 e pós-intervenção, encontrou-se diferença estatística significativa em todas as escalas, bem como no escore total, em direção compatível com um efeito benéfico da intervenção. 
Tabela 1 . Escores médios na avaliação sociocognitiva e resultados das comparações entre avaliações pré e pós-intervenção

\begin{tabular}{|c|c|c|c|c|c|c|c|c|}
\hline \multirow[b]{2}{*}{ Componente } & \multicolumn{3}{|c|}{$\begin{array}{c}\text { GI - 2010 } \\
(\mathrm{n}=\mathbf{2 5})\end{array}$} & \multicolumn{5}{|c|}{$\begin{array}{c}\text { GII - } 2011 \\
(\mathrm{n}=\mathbf{2 0})\end{array}$} \\
\hline & $\begin{array}{c}\text { Pré- } \\
\text { Intervenção } \\
\text { M(EP) }\end{array}$ & $\begin{array}{l}\text { Pós- } \\
\text { Intervenção } \\
\text { M(EP) }\end{array}$ & $t_{\text {pré-pós }}$ & $\begin{array}{c}\text { Pré- } \\
\text { Intervenção } \\
1 \mathrm{M}(\mathrm{EP})\end{array}$ & $\begin{array}{c}\text { Pré- } \\
\text { Intervenção } \\
2 \mathrm{M}(\mathrm{EP})\end{array}$ & $t_{\text {pré1-pré2 }}$ & $\begin{array}{c}\text { Pós- } \\
\text { Intervenção } \\
\text { M(EP) }\end{array}$ & $t_{\text {pré2-pós }}$ \\
\hline O que está acontecendo? & $61,80(1,94)$ & $84,36(1,94)$ & $-13,34 * *$ & $58,40(2,17)$ & $68,05(2,17)$ & $-5,10 * *$ & $84,38(2,20)$ & $-8,48 * *$ \\
\hline $\begin{array}{l}\text { O que os personagens estão pensando } \\
\text { e sentindo? }\end{array}$ & $72,16(2,74)$ & $99,64(2,74)$ & $-10,44 * *$ & $63,95(3,06)$ & $72,30(3,06)$ & $-2,84 * *$ & $99,24(3,11)$ & $-9,00 * *$ \\
\hline Quais as metas e intenções? & $35,20(1,20)$ & $44,60(1,20)$ & $-8,72 * *$ & $33,80(1,34)$ & $35,95(1,34)$ & $-1,78$ & $44,70(1,36)$ & $-7,13 * *$ \\
\hline $\begin{array}{l}\text { O que os personagens alcançam com } \\
\text { suas ações? }\end{array}$ & $15,88(0,79)$ & $22,28(0,79)$ & $-9,84 * *$ & $14,90(0,88)$ & $16,35(0,88)$ & $-1,99$ & $22,42(0,89)$ & $-8,20 * *$ \\
\hline $\begin{array}{l}\text { Como ...executam e monitoram } \\
\text { comportamentos? }\end{array}$ & $31,32(1,30)$ & $43,96(1,30)$ & $-9,98 * *$ & $30,30(1,46)$ & $32,45(1,46)$ & $-1,52$ & $45,11(1,48)$ & $-8,79 * *$ \\
\hline Quais as lições aprendidas? & $4,52(0,40)$ & $7,36(0,40)$ & $-6,34 * *$ & $3,35(0,44)$ & $3,35(0,44)$ & 0 & $7,31(0,45)$ & $-7,77 * *$ \\
\hline Total & $220,88(6,84)$ & $302,20(6,84)$ & $-17,55 * *$ & $204,70(7,65)$ & $228,45(7,65)$ & $-4,58 * *$ & $303,23(7,71)$ & $-14,17 * *$ \\
\hline
\end{tabular}

Tabela 2 . Escores médios no QRSH-RP e resultados das comparações entre avaliações pré e pós-intervenção

\begin{tabular}{|c|c|c|c|c|c|c|c|c|}
\hline \multirow[b]{2}{*}{ Componente } & \multicolumn{3}{|c|}{$\begin{array}{c}\text { GI - } 2010 \\
(n=25)\end{array}$} & \multicolumn{5}{|c|}{$\begin{array}{c}\text { GII - } 2011 \\
(\mathrm{n}=\mathbf{2 0})\end{array}$} \\
\hline & $\begin{array}{c}\text { Pré- } \\
\text { Intervenção } \\
\mathrm{M}(\mathrm{EP})\end{array}$ & $\begin{array}{l}\text { Pós- } \\
\text { Intervenção } \\
\text { M(EP) }\end{array}$ & $t_{\text {pré-pós }}$ & $\begin{array}{c}\text { Pré- } \\
\text { Intervenção } \\
1 \mathrm{M}(\mathrm{EP})\end{array}$ & $\begin{array}{c}\text { Pré- } \\
\text { Intervenção } \\
2 \mathrm{M}(\mathrm{EP})\end{array}$ & $t_{\text {prél-pré2 }}$ & $\begin{array}{l}\text { Pós- } \\
\text { Intervenção } \\
M(E P)\end{array}$ & $t_{\text {pré2-pós }}$ \\
\hline Sociab. e Expr. Emocional & $21,04(1,02)$ & $24,52(1,02)$ & $-3,88 * *$ & $21,20(1,14)$ & $21,45(1,14)$ & $-0,25$ & $26,65(1,15)$ & $-5,09 * *$ \\
\hline Iniciativa Social & $6,88(0,71)$ & $8,88(0,71)$ & $-3,29 * *$ & $6,90(0,79)$ & $8,55(0,79)$ & $-2,43 *$ & $11,02(0,80)$ & $-3,57 * *$ \\
\hline Busca por Suporte & $4,92(0,24)$ & $5,48(0,24)$ & $-2,69 * *$ & $4,90(0,27)$ & $4,75(0,27)$ & 0,65 & $5,71(0,28)$ & $-4,06 * *$ \\
\hline Total & $34,08(1,75)$ & $40,44(1,75)$ & $-4,41 * *$ & $34,20(1,96)$ & $35,95(1,96)$ & $-1,09$ & $45,31(1,98)$ & $-5,71 * *$ \\
\hline
\end{tabular}

Tabela 3. Escores médios no SDQ e resultados das comparações entre avaliações pré e pós-intervenção

\begin{tabular}{|c|c|c|c|c|c|c|c|c|}
\hline \multirow[b]{2}{*}{ Componente } & \multicolumn{3}{|c|}{$\begin{array}{c}\text { GI - } 2010 \\
(n=25)\end{array}$} & \multicolumn{5}{|c|}{$\begin{array}{c}\text { GII - 2011 } \\
(\mathrm{n}=\mathbf{2 0})\end{array}$} \\
\hline & $\begin{array}{c}\text { Pré- } \\
\text { Intervenção } \\
\text { M(EP) }\end{array}$ & $\begin{array}{c}\text { Pós- } \\
\text { Intervenção } \\
\text { M(EP) }\end{array}$ & $t_{\text {pré-pós }}$ & $\begin{array}{c}\text { Pré- } \\
\text { Intervenção } \\
1 \mathrm{M}(\mathrm{EP})\end{array}$ & $\begin{array}{c}\text { Pré- } \\
\text { Intervenção } \\
2 \mathrm{M}(\mathrm{EP})\end{array}$ & $t_{\text {prél-pré2 }}$ & $\begin{array}{l}\text { Pós- } \\
\text { Intervenção } \\
\text { M(EP) }\end{array}$ & $t_{\text {pré2-pós }}$ \\
\hline Sintomas Emocionais & $2,68(0,34)$ & $2,48(0,34)$ & 0,49 & $2,60(0,38)$ & $2,70(0,38)$ & $-0,22$ & $1,55(0,39)$ & $2,50^{*}$ \\
\hline Problemas de Conduta & $2,16(0,53)$ & $1,64(0,53)$ & 1,13 & $2,95(0,59)$ & $2,85(0,59)$ & 0,19 & $1,09(0,60)$ & $3,36^{* *}$ \\
\hline Hiperatividade & $4,48(0,57)$ & $3,04(0,57)$ & $3,22 * *$ & $4,95(0,64)$ & $5,00(0,64)$ & $-0,10$ & $2,56(0,65)$ & $4,8^{* *}$ \\
\hline Problemas de Relacionamento & $1,32(0,23)$ & $0,68(0,23)$ & $2,80 * *$ & $1,55(0,26)$ & $1,50(0,26)$ & 0,20 & $0,42(0,27)$ & $4,17 * *$ \\
\hline Comportamento Prossocial & $6,72(0,49)$ & $8,48(0,49)$ & $-3,68 * *$ & $6,45(0,55)$ & $7,45(0,55)$ & $-1,87$ & $9,50(0,56)$ & $-3,76 * *$ \\
\hline Total & $10,64(1,22)$ & $7,84(1,22)$ & $2,8^{* *}$ & $12,05(1,37)$ & $12,05(1,37)$ & 0 & $5,59(1,39)$ & $5,68^{* *}$ \\
\hline
\end{tabular}

Nota. $^{*} p<0,05 ; * * p<0,01$

\section{Discussão}

Os resultados obtidos no presente estudo vão ao encontro dos achados das investigações que visam à promoção sociocognitiva (Berlin et al., 2011; Borges \& Marturano 2009; Fraser et al., 2005; Rodrigues et al., 2010, Rodrigues, 2014). Sugere-se que, à medida que as crianças aprimoram suas habilidades sociocognitivas com a exposição ao programa, expandem, também, suas habilidades sociais, ampliam os comportamentos prossociais e reduzem os problemas de comportamento. Tais achados se alinham à observação prévia de que crianças mais habilidosas socialmente tendem a apresentar menos problemas de relacionamento (Del Prette \& Del Prette, 2005; Fenning, Bake, \& Juvonen, 2011; Helmsen et al., 2012). Fraser et al. (2005) após implementarem um programa de resolução de problemas interpessoais, baseado nos passos do modelo de Dodge e Crick (1994), encontraram resultados que convergiram com os evidenciados na presente investigação: as crianças participantes do programa aprimoraram o relacionamento interpessoal e se mostraram mais ajustadas socialmente.

Quanto aos resultados da investigação sociocognitiva referente ao aprimoramento das habilidades, independentes da intervenção, inseridas nos componentes $O$ que os personagens alcançam com suas ações?, O que os personagens estão pensando e sentindo? e O que está acontecendo? poderiam ser atribuídos às experiências desenvolvimentais infantis. Rodrigues (2008) ressalta que as experiências familiares e educacionais contribuem para 
a ampliação da decodificação de pistas sociais. Tem-se, então, um aprimoramento natural no desenvolvimento no que tange à identificação das pistas. Yamaguchi et al. (2009) consideram, também, o aprimoramento das habilidades sociocognitivas decorrentes do desenvolvimento.

Sugere-se, ainda, que a mera exposição à avaliação sociocognitiva, que conta com a leitura do livro Anjinho, tenha ocasionado uma aprendizagem em relação aos acontecimentos da história, de forma que a segunda avaliação, denominada de pré-intervenção 2 , feita com as crianças do GII evocou memórias registradas na primeira avaliação. Dessa maneira, o aprimoramento na decodificação de pistas sociais pode ser associado ao fato de as crianças já conhecerem a história. O achado vai ao encontro dos resultados obtidos por Rohrer, Taylor e Sholar (2010). Os autores investigaram o efeito da aprendizagem decorrente da avaliação e identificaram nas crianças um melhor desempenho no teste decorrente da mera repetição avaliativa (Rohrer et al., 2010).

Observa-se, também, nos resultados sociocognitivos, que os ganhos nos componentes acima discutidos foram mais expressivos depois da intervenção, em ambos os grupos, do que entre a pré-intervenção 1 e a pré-intervenção 2, no GII. Portanto, apesar de as crianças apresentarem aprimoramento nesse componente independente da intervenção, sugerese que, por meio do programa de histórias infantis, os incrementos são mais evidenciados. Segundo Rodrigues et al. (2007), as histórias infantis constituem um excelente recurso para trabalhar os sentimentos, uma vez que os livros são ricos em imagens e palavras que descrevem as emoções dos personagens.

Parece, então, que a estrutura das narrativas infantis possibilita a exploração e a discussão dos aspectos relevantes ao processamento da informação social (Rodrigues \& Ribeiro, 2011; Teglasi \& Rothman, 2001). As crianças de educação infantil apresentaram ganhos sociocognitivos; desenvolveram e aprimoraram a compreensão acerca das emoções, pensamentos, intenções, metas e comportamento dos personagens, desenvolvendo e refinando, portanto, a cognição social.

Para o indicador das habilidades sociais, os resultados obtidos no escore total do instrumento QRSH-RP e nos fatores Sociabilidade e Expressividade Emocional e Busca por Suporte sugerem incrementos nos comportamentos sociais infantis advindos da intervenção. Tais resultados vão ao encontro daqueles encontrados por Borges e Marturano (2009) e Rodrigues et al. (2010). Os referidos estudos, objetivando a promoção das habilidades de solução de problemas interpessoais em uma abordagem sociocognitiva, resultaram em crianças com um aprimorado desempenho prossocial, no sentido de que os participantes se relacionaram mais e melhor, confiaram mais nos colegas e emitiram mais comportamentos generosos depois da intervenção.

O programa de histórias infantis não trabalhou diretamente a promoção das habilidades sociais, dessa forma pode-se supor que os benefícios encontrados possam ser devidos ao progresso nas habilidades sociocognitivas. Tal interpretação está de acordo com a afirmativa de Rodrigues (2008). A autora aponta que habilidades sociocognitivas podem ser consideradas mediadoras do desempenho social: crianças mais habilidosas do ponto de vista sociocognitivo tendem a emitir comportamentos mais habilidosos socialmente.

Os resultados das habilidades sociais também corroboram aqueles encontrados por Mayeux e Cillessen (2003): os autores apontam que crianças que apresentam um desempenho competente em cada passo do modelo de processamento da informação social são também mais competentes nas relações com os pares. Kalyva e Agaliotis (2009) apontam, também, que as histórias contribuem para o ensino das habilidades sociais e levam a uma maior compreensão da perspectiva do outro.

Considerando os resultados da pesquisa com intervenção realizada por Rodrigues e Ribeiro (2011), encontram-se mais indícios de que a promoção das habilidades sociocognitivas impacta as habilidades sociais. Ao constatarem, nos participantes da intervenção, discreta superioridade de respostas empáticas sobre o grupo não participante (respectivamente, 65\% e 55\%), as referidas autoras apontam que um programa dirigido a fomentar o desenvolvimento sociocognitivo, por meio da mediação dialogada das histórias infantis, tende a viabilizar o aprimoramento das habilidades empáticas.

O fator Busca por Suporte apresentou aumento significativo após a intervenção, em ambos os grupos, sugerindo, então, que as crianças após o programa procuraram mais a atenção dos professores. O fator em questão é discutido na literatura como uma dimensão que tende a diminuir de importância no repertório de habilidades sociais, conforme a criança desenvolve outros recursos para lidar de forma independente com os desafios do seu dia a dia (Bolsoni-Silva et al., 2009b). As crianças, à medida que refinam tais recursos, diminuem a busca por ajuda, atenção e informações, ganhando autonomia para buscar o que é necessário. Estudos empíricos detectaram declínio na estratégia de coping $^{2}$ baseada na busca pelo suporte do adulto, entre 5 e 7 anos de idade (Skinner \& Zimmer-Gembeck, 2007). No contexto dos resultados encontrados, o programa de intervenção afetou as crianças aparentemente em direção oposta a essa tendência desenvolvimental apontada na literatura. Sugere-se que a contraposição é apenas aparente, visto que a busca pelo suporte do adulto é estratégia de solução de problemas típica da faixa de desenvolvimento das crianças participantes do presente estudo; assim sendo, o programa pode ter oportunizado o exercício da estratégia, sem necessariamente afetar seu curso futuro. Em outras palavras, sugere-se que com a exposição ao programa as crianças se tornaram mais habilidosas para a busca por ajuda, informação e atenção da professora; dando-se esse progresso em um contexto de acolhimento por parte das educadoras, as duas condições - exposição ao programa e acolhimento da professora - podem ter-se conjugado para que, no presente estudo, obtivesse-se aumento na busca do apoio do adulto, o que não se contrapõe necessariamente à tendência evolutiva de redução à medida que as crianças se tornam mais velhas, conforme observado por outros autores (Bolsoni-Silva,

2 Estratégias para administrar demandas externas e internas, notadas pela criança como estressantes e avaliadas como sobrecarga aos recursos pessoais (Bolsoni-Silva et al., 2009b). 
Marturano \& Freiria, 2009; Skinner \& Zimmer-Gembeck, 2007).

No detalhamento das análises das capacidades e dificuldades infantis, tem-se uma redução significativa do indicador hiperatividade após a intervenção. Os achados se alinham àqueles encontrados por Fraser et al. (2005) e Teglasi e Rothman (2001). Intervenções que visam ao aprimoramento do processamento da informação social contribuem para a capacidade de refletir antes de agir, reduzindo, assim, a impulsividade. Os demais dados significativos, redução dos problemas de comportamento bem como no escore total e aumento do comportamento prossocial, alinham-se aos resultados apresentados pelo instrumento QRSH-RP.

A proposta de intervenção do presente estudo, baseada no processamento da informação social, visa à promoção de habilidades sociocognitivas que favoreçam a criança lidar com demandas sociais. Observa-se, portanto, uma particularidade do programa de histórias, já que a intervenção não foi efetiva para reduzir sintomas emocionais e problemas de conduta, porém foi efetiva no aprimoramento dos comportamentos prossociais e na redução dos problemas de relacionamento e hiperatividade. Assim, é possível sugerir que os achados validam a abordagem teórica no sentido da especificidade da intervenção, já que a configuração dos resultados referentes ao SDQ mostra, com clareza, que os benefícios da intervenção incidiram predominantemente sobre comportamentos interpessoais e sobre a capacidade de autorregulação da atenção.

Ambos os grupos mostraram resposta positiva ao programa, porém com particularidades. Os participantes de GII, que passaram pela primeira sondagem sociocognitiva em idade mais jovem que os de GI e repetiram essa avaliação antes de iniciar o programa, mostraram: (a) progresso independente da intervenção em alguns componentes sociocognitivos; (b) aumento nas habilidades de Iniciativa Social antes da intervenção; (c) progresso significativamente maior que o de GI na Iniciativa Social, após a intervenção. Esses resultados são sugestivos de que o desempenho mais competente nos passos do processamento da informação social, instigado pela repetição da investigação sociocognitiva, pode ter levado a um aprimoramento nas habilidades de iniciativa social, potencializando os efeitos da intervenção sobre essas habilidades. Obviamente, essa é uma interpretação sujeita à verificação empírica.

As estratégias de trabalho que impactam toda uma turma no que tange à melhora do relacionamento interpessoal são bastante valorizadas na literatura (Borges \& Marturano, 2009; Brownell et al., 2008; Del Prette \& Del Prette, 2005; Lansford et al., 2011; Rodrigues et al., 2010; Rodrigues \& Ribeiro, 2011; Teglasi \& Rothman, 2001; Thomas et al., 2011). Além disso, as instituições educacionais demandam estratégias que visem à promoção do bem-estar infantil e ao desenvolvimento de competências. A escola, por envolver aspectos inerentes à socialização, é um local privilegiado para o desenvolvimento desse tipo de trabalho.

O programa desenvolvido, com base na leitura de histórias infantis, apresentou, portanto, resultados promissores para a área das habilidades sociais e da cognição social; sendo assim, pode contribuir para a promoção de desenvolvimento em contexto escolar. Nesse sentido, seus resultados abrem perspectivas para novas investigações. Seria importante verificar, por meio de intervenção com estudo de seguimento, por que extensão de tempo os resultados se mantêm. Pesquisas com foco no treinamento de professores poderiam dar subsídios para a organização de cursos de formação, a fim de suprir lacunas na formação dos educadores, no que tange a dificuldades para lidar com os problemas de relacionamento entre crianças pequenas.

O estudo apresenta limitações que devem ser levadas em consideração. Uma das limitações relaciona-se ao instrumento de avaliação sociocognitiva. O instrumento, apesar de aprimorado e sistematizado para o uso na pesquisa, ainda carece de validação. Outra limitação diz respeito ao fato de a intervenção e a avaliação sociocognitiva terem sido conduzidas pela mesma pessoa; ainda que tenha favorecido a expressão livre das crianças diante de um adulto bastante familiar, esse não é procedimento tido como ideal em pesquisas de avaliação da efetividade de intervenções.

Tem-se, ainda, as habilidades sociais e as capacidades e dificuldades infantis avaliadas somente pelas professoras. $\mathrm{O}$ estudo teria sido enriquecido com a autoavaliação das crianças ou o julgamento pelos pares, mediante o uso de instrumentos apropriados à faixa etária dos participantes. Além do limite imposto por uma única fonte de informação, há também a circunstância de que as professoras estavam cientes da participação das crianças no programa de intervenção, o que pode ter introduzido um viés positivo nas avaliações pós-intervenção.

Apesar das limitações, os resultados encontrados não são invalidados. Ao contrário, da forma como a pesquisa foi implementada, em condições de vida real no dia a dia de uma escola de educação infantil, atendeu-se ao requisito de validade ecológica, tão importante para fundamentar práticas baseadas em evidência. O programa de histórias infantis mostra-se, então, promissor para ser aplicado em contexto escolar a fim de promover desenvolvimento nas crianças, ampliando seus recursos para lidar com os desafios da convivência em ambientes coletivos.

\section{Referências}

Achenbach, T. M., \& Edelbrock, C. S. (1979). The child behavior profile: II. Boys aged 12-16 and girls aged 6-11 and 12-16. Journal of Consulting and Clinical Psychology, 47(2), 223-233.

Berlin, L. J., Dunning, R. D., \& Dodge, K. A. (2011). Enhancing the transition to kindergarten: A randomized trial to test the efficacy of the "Stars" summer kindergarten orientation program. Early Childhood Research Quarterly, 26, 247-254.

Bolsoni-Silva, A. T., Marturano, E. M., \& Loureiro, S. R. (2009a). Construction and Validation of the Brazilian Questionário de Respostas Socialmente Habilidosas Segundo Relato de Professores (QRSH-RP). The Spanish Journal of Psychology, 12(1), 349-359.

Bolsoni-Silva, A. T., Marturano, E. M., \& Freiria, R. L. B. (2009b). Indicativos de problemas de comportamento e de habilidades sociais em crianças: Um estudo longitudinal. Psicologia: Reflexão e Crítica, 23(3), 506-515. 
Borges, D. S. C., \& Marturano, E. M. (2009). Aprendendo a gerenciar conflitos: Um programa de intervenção para a $1^{\mathrm{a}}$ série do ensino fundamental. Paidéia, 19(42), 17-26.

Bornstein, M. H., Hahn, C. S., \& Haynes, O. M. (2010). Social competence, externalizing, and internalizing behavioral adjustment from early childhood through early adolescence: Developmental cascades. Development and Psychopathology, 22(4), 717-735.

Brownell, C., Belsky, J., Booth-LaForce, C., Bradley, R., Campbell, S. B, Clarke-Stewart, K. A., \& Cox, M. (2008). Social competence with peers in third grade: Associations with earlier peer experiences in childcare. Social Development, 17, 419-453.

Burt, K. B., \& Roisman, G. I. (2010). Competence and psychopathology: Cascade effects in the NICHD Study of Early Child Care and Youth Development. Development and Psychopathology, 22, 557-567.

Del Prette, Z. A. P., \& Del Prette, A. (2005). Psicologia das habilidades sociais na infância: Teoria e prática. Petrópolis, SP: Vozes.

Dias, J. P. (2012). Literatura e desenvolvimento sociocognitivo: Avaliação e implementação de um programa na educação infantil (Dissertação de Mestrado). Faculdade de Filosofia Ciências e Letras de Ribeirão Preto, Universidade de São Paulo, São Paulo.

Dodge, K. A. (1986). A social information processing model of social competence in children. In M. Perlmutter (Org.), Minnesota symposium in child psychology. Cognitive perspectives in children's social and behavioral development (pp.77-125). Hillsdale, New Jersey: Erlbaum.

Dodge, K. A., \& Crick, N. R. (1994). A review and reformulation of social information-processing mechanisms in children's social adjustment. Psychological Bulletin, 115(1), 74-101.

Fenning, R. M., Bake, B. L., \& Juvonen, J. (2011). Emotion discourse, social cognition, and social skills in children with and without developmental delays. Child Development, 82(2), 717-731.

Fleitlich, B., Cortázar, P. G., \& Goodman, R. (2000). Questionário de Capacidades e Dificuldades (SDQ). Infanto - Revista de Neuropsiquiatria da Infância e Adolescência, 8, 44-50.

Fraser, M. W., Galinsky, M. J., Smokowski, P. R., Day, S. H., Terzian, M. A., Rose, R. A., \& Guo, S. (2005). Social informationprocessing skills training to promote social competence and prevent aggressive behavior in the third grade. Journal of Consulting and Clinical Psychology, 73(6), 1045-1055.

Furnari, E. (2004). Anjinho. São Paulo: Ática.

Goodman, R (1997). The Strengths and Difficulties Questionnaire: A research note. Journal of Child Psychology and Psychiatry, 38, 581-586.

Helmsen, J., Koglin, U., \& Petermann, F. (2012). Emotion regulation and aggressive behavior in preschoolers: The mediating role of social information processing. Child Psychiatry \& Human Development, 43, 87-101.

Kalyva, E., \& Agaliotis, I. (2009). Can social stories enhance the interpersonal conflict resolution skills of children with LD? Research in Developmental Disabilities, 30, 192-202.
Lansford, J. E. , Malone, P. S., Dodge, K. A., Crozier, J. C., Pettit, G. S., \& Bates, J. E. (2006). A 12-Year prospective study of patterns of social information processing problems and externalizing behaviors. Journal of Abnormal Child Psychology, 34, 715-724.

Mayeux, L., \& Cillessen, A. H. (2003). Development of social problem solving in early childhood: Stability, change, and associations with social competence. Journal of Genetic Psychology, 164, 153-173.

Rodrigues, M. C., \& Ribeiro, N. N. (2011). Avaliação da empatia em crianças participantes e não participantes de um programa para desenvolvimento sociocognitivo. Psicologia: Teoria e Prática, 13(2), 114-126.

Rodrigues, M. C. (2008). Leitura de histórias e sua importância no desenvolvimento infantil. In A. J. G. Barbosa, L. M. Lourenço \& M. M. P. E. Mota (Orgs.), Desenvolvimento psicossocial: Temas em educação e saúde (pp. 19-31). Campinas: Alínea.

Rodrigues, M. C. (2014). Leitura mediada com enfoque sociocognitivo: Subsídios teórico-práticos. In A. Roazzi \& T. Sperb (Orgs.), O desenvolvimento de competências sociocognitivas: Novas perspectivas (pp. 63-93). São Paulo: Vetor Editora.

Rodrigues, M. C., Oliveira, P. A., Rubac, J. S., \& Tavares, A. L. (2007). Literatura infantil, teoria da mente e processamento de informação social. Psicologia Escolar Educacional, 11(1), 77-88.

Rodrigues, M. C., Dias, J. P., \& Freitas, M. R. L. (2010). Resolução de problemas interpessoais: Promovendo o desenvolvimento sociocognitivo na escola. Psicologia em Estudo, 15(4), 831839.

Rohrer, D., Taylor, K., \& Sholar, B. (2010). Tests enhance the transfer of learning. Journal of Experimental Psychology: Learning, Memory, and Cognition, 36(1), 233-239.

Schall, R. (1991). Estimation in generalized linear models with random effects. Biometrika, 78(4), 719-727.

Silver, R. B., Measelle, J. R., Armstrong, J. M., \& Essex, M. J. (2010). The impact of parents, child care providers, teachers, and peers on early externalizing trajectories. Journal of School Psychology, 48(6), 555-583.

Teglasi, H., \& Rothman, L. (2001). Stories: A classroom-based program to reduce aggressive behavior. Journal of School Psychology, 39(1), 71-94.

Thomas, R.E., Bierman, K.L., \& Powers, C.J. (2011). The influence of classroom aggression and classroom climate on the early development of aggressive-disruptive behavior problems in school. Child Development, 82, 751-757.

Vasconcellos, S. J. L., Picon, P., Prochnow, L. P., \& Gauer, G. J. C. (2006). O processamento das informações sociais em crianças e adolescentes agressivos. Estudos de Psicologia, 11(3), 275-279.

Yamaguchi, M., Kuhlmeier, V. A., Wynn, K., \& van Marle, K. (2009). Continuity in social cognition from infancy to childhood. Developmental Science, 12(5), 746-752

Recebido em 09.04.2013

Primeira decisão editorial em 17.03.2015

Versão final em 02.04.2015

Aceito em 05.06.2015 